Commun. Korean Math. Soc. 27 (2012), No. 1, pp. 57-68

http://dx.doi.org/10.4134/CKMS.2012.27.1.057

\title{
ON COMMUTING GRAPHS OF GROUP RING $Z_{n} Q_{8}$
}

\author{
Jianlong Chen, Yanyan GaO, and Gaohua Tang
}

\begin{abstract}
The commuting graph of an arbitrary ring $R$, denoted by $\Gamma(R)$, is a graph whose vertices are all non-central elements of $R$, and two distinct vertices $a$ and $b$ are adjacent if and only if $a b=b a$. In this paper, we investigate the connectivity, the diameter, the maximum degree and the minimum degree of the commuting graph of group ring $Z_{n} Q_{8}$. The main result is that $\Gamma\left(Z_{n} Q_{8}\right)$ is connected if and only if $n$ is not a prime. If $\Gamma\left(Z_{n} Q_{8}\right)$ is connected, then $\operatorname{diam}\left(Z_{n} Q_{8}\right)=3$, while $\Gamma\left(Z_{n} Q_{8}\right)$ is disconnected then every connected component of $\Gamma\left(Z_{n} Q_{8}\right)$ must be a complete graph with a same size. Further, we obtain the degree of every vertex in $\Gamma\left(Z_{n} Q_{8}\right)$, the maximum degree and the minimum degree of $\Gamma\left(Z_{n} Q_{8}\right)$.
\end{abstract}

\section{Introduction}

Let $G$ be a group and $R$ a ring. We denote $R G$ by the set of all formal linear combinations of the forms $\alpha=\sum_{g \in G} a_{g} g$, where $a_{g} \in R$ and $a_{g}=0$ almost everywhere, that is, only a finite number of coefficients are different from 0 in each of these sums. Notice that it follows from our definition that given two elements, $\alpha=\sum_{g \in G} a_{g} g$ and $\beta=\sum_{g \in G} b_{g} g \in R G$, we have that $\alpha=\beta$ if and only if $a_{g}=b_{g}, \forall g \in G$. We define the sum of two elements in $R G$ componentwise:

$$
\left(\sum_{g \in G} a_{g} g\right)+\left(\sum_{g \in G} b_{g} g\right)=\sum_{g \in G}\left(a_{g}+b_{g}\right) g .
$$

Also, given two elements $\alpha=\sum_{g \in G} a_{g} g$ and $\beta=\sum_{h \in G} b_{h} h \in R G$ we define their product by

$$
\alpha \beta=\sum_{g, h \in G} a_{g} b_{h} g h .
$$

The commuting graph of an arbitrary ring $R$ denoted by $\Gamma(R)$, is a graph with vertex set $R \backslash \mathcal{Z}(R)$, where $\mathcal{Z}(R)$ is the center of $R$, and two distinct

Received September 21, 2010.

2010 Mathematics Subject Classification. 16S34, 20C05, 05C12, 05C40.

Key words and phrases. group ring, commuting graph, connected component, diameter of a graph. 
vertices $a$ and $b$ are adjacent if and only if $a b=b a$. In 2004, the notion of commuting graph of a ring was first introduced by Akbari, Ghandehari, Hadian and Mohammadian in [2]. The commuting graphs of semisimple rings have been studied in $[1,2,4,3]$. And in this paper, we investigate some properties of $\Gamma\left(Z_{n} Q_{8}\right)$, where $Z_{n} Q_{8}=\left\{x_{1}+x_{2} a+x_{3} a^{2}+x_{4} a^{3}+x_{5} b+x_{6} a b+x_{7} a^{2} b+x_{8} a^{3} b \mid\right.$ $\left.x_{i} \in Z_{n}, i=1,2, \ldots, 8\right\}$ and $Z_{n}=\{0,1, \ldots, n-1\}$ is the module $n$ residue class ring, $Q_{8}=\left\langle a, b \mid a^{4}=1, b^{2}=1, a b=b a^{-1}\right\rangle=\left\{1, a, a^{2}, a^{3}, b, a b, a^{2} b, a^{3} b\right\}$ is the quaternion group.

Let $R$ be a ring and $R^{*}=R \backslash\{0\}$. Given integers $a$ and $b$, we denote by $(a, b)$ the greatest common divisor of $a$ and $b$. If $p$ is a prime and $t$ is a nonnegative integer, then we use the notation $p^{t} \| a$ to mean that $p^{t} \mid a$ and $p^{t+1} \nmid a$. The ring of $n$ by $n$ full matrices over a ring $R$ is denoted by $M_{n}(R)$.

In this paper, all graphs are simple and undirected and $|G|$ denotes the number of vertices of the graph $G$. In a graph $G$, the degree of a vertex $v$ is denoted by $d(v)$. And the minimum degree and maximum degree of $G$ are denoted by $\delta(G)$ and $\triangle(G)$, respectively. A path of length $r$ from a vertex $x$ to another vertex $y$ in $G$ is a sequence of $r+1$ distinct vertices starting with $x$ and ending with $y$ such that consecutive vertices are adjacent. For a connected graph $H$, the diameter of $H$ is denoted by $\operatorname{diam}(H)$. An induced subgraph of $G$ that is maximal, subject to being connected, is called a connected component of $G$.

In this paper, we investigate the connectivity, the diameter, the maximum degree and the minimum degree of the commuting graph of group $\operatorname{ring} Z_{n} Q_{8}$. In Section 2, we show that $\Gamma\left(Z_{n} Q_{8}\right)$ is connected if and only if $n$ is not a prime. If $\Gamma\left(Z_{n} Q_{8}\right)$ is connected, then $\operatorname{diam}\left(Z_{n} Q_{8}\right)=3$, while $\Gamma\left(Z_{n} Q_{8}\right)$ is disconnected then every connected component of $\Gamma\left(Z_{n} Q_{8}\right)$ must be a complete graph with a same size. In Section 3, we obtain the degree of every vertex in $\Gamma\left(Z_{n} Q_{8}\right)$, the maximum degree and the minimum degree of $\Gamma\left(Z_{n} Q_{8}\right)$.

\section{The connectivity and diameter of $\Gamma\left(Z_{n} Q_{8}\right)$}

Lemma 2.1 ([2, Theorem 2]). If $F$ is a finite field, then $\Gamma\left(M_{2}(F)\right)$ is a graph with $|F|^{2}+|F|+1$ connected components of size $|F|^{2}-|F|$ which each of them is a complete graph.

Lemma 2.2. Let $n$ be an arbitrary positive integer. Then $\mathcal{Z}\left(Z_{n} Q_{8}\right)=\{\alpha=$ $\left.x_{1}+x_{2} a+x_{3} a^{2}+x_{2} a^{3}+x_{5} b+x_{6} a b+x_{5} a^{2} b+x_{6} a^{3} b \mid x_{1}, x_{2}, x_{3}, x_{5}, x_{6} \in Z_{n}\right\}$, $\left|\mathcal{Z}\left(Z_{n} Q_{8}\right)\right|=n^{5}$ and $\left|\Gamma\left(Z_{n} Q_{8}\right)\right|=n^{8}-n^{5}$, where $\mathcal{Z}\left(Z_{n} Q_{8}\right)$ denotes the center of the group ring $Z_{n} Q_{8}$.

Proof. $\forall \alpha=x_{1}+x_{2} a+x_{3} a^{2}+x_{4} a^{3}+x_{5} b+x_{6} a b+x_{7} a^{2} b+x_{8} a^{3} b, \beta=$ $y_{1}+y_{2} a+y_{3} a^{2}+y_{4} a^{3}+y_{5} b+y_{6} a b+y_{7} a^{2} b+y_{8} a^{3} b \in \Gamma\left(Z_{n} Q_{8}\right)$, we have 
$\alpha \beta=\beta \alpha$ if and only if the following system of congruence equations $(*)$ holds.

$\left.{ }^{\prime}\right)\left\{\begin{array}{l}\left(x_{6}-x_{8}\right) y_{5}-\left(x_{5}-x_{7}\right) y_{6}-\left(x_{6}-x_{8}\right) y_{7}+\left(x_{5}-x_{7}\right) y_{8} \equiv 0 \quad(\bmod n)(1) \\ \left(x_{6}-x_{8}\right) y_{2}-\left(x_{6}-x_{8}\right) y_{4}-\left(x_{2}-x_{4}\right) y_{6}+\left(x_{2}-x_{4}\right) y_{8} \equiv 0 \quad(\bmod n)(2) \\ \left(x_{5}-x_{7}\right) y_{2}-\left(x_{5}-x_{7}\right) y_{4}-\left(x_{2}-x_{4}\right) y_{5}+\left(x_{2}-x_{4}\right) y_{7} \equiv 0 \quad(\bmod n)(3)\end{array}\right.$

Suppose that $\alpha=x_{1}+x_{2} a+x_{3} a^{2}+x_{4} a^{3}+x_{5} b+x_{6} a b+x_{7} a^{2} b+x_{8} a^{3} b \in$ $\mathcal{Z}\left(Z_{n} Q_{8}\right)$, then it is clear that $a \alpha=\alpha a$. Thus by the system $(*)$, it follows that

$$
\begin{cases}x_{6}-x_{8} \equiv 0 & (\bmod n) \\ x_{5}-x_{7} \equiv 0 & (\bmod n)\end{cases}
$$

i.e., $x_{6} \equiv x_{8}(\bmod n)$, and $x_{5} \equiv x_{7}(\bmod n)$.

In addition, we also have $b \alpha=\alpha b$, hence we have that

$$
\begin{cases}x_{6}-x_{8} \equiv 0 & (\bmod n) \\ x_{2}-x_{4} \equiv 0 & (\bmod n)\end{cases}
$$

i.e., $x_{6} \equiv x_{8}(\bmod n)$, and $x_{2} \equiv x_{4}(\bmod n)$.

Therefore, we have $x_{2} \equiv x_{4}(\bmod n), x_{5} \equiv x_{7}(\bmod n)$ and $x_{6} \equiv x_{8} \quad(\bmod$ $n$ ). Hence, $\alpha=x_{1}+x_{2} a+x_{3} a^{2}+x_{2} a^{3}+x_{5} b+x_{6} a b+x_{5} a^{2} b+x_{6} a^{3} b$ and it is easy to verify that such $\alpha$ is in the center of $Z_{n} Q_{8}$.

Thus $\mathcal{Z}\left(Z_{n} Q_{8}\right)=\left\{\alpha=x_{1}+x_{2} a+x_{3} a^{2}+x_{2} a^{3}+x_{5} b+x_{6} a b+x_{5} a^{2} b+x_{6} a^{3} b \mid\right.$ $\left.x_{1}, x_{2}, x_{3}, x_{5}, x_{6} \in Z_{n}\right\}$ and $\left|\mathcal{Z}\left(Z_{n} Q_{8}\right)\right|=n^{5},\left|\Gamma\left(Z_{n} Q_{8}\right)\right|=n^{8}-n^{5}$.

Theorem 2.3. Suppose $n=p^{t}$, where $p \geq 2$ is a prime and $t \geq 2$. Then $\Gamma\left(Z_{n} Q_{8}\right)$ is a connected graph and $\operatorname{diam}\left(\Gamma\left(Z_{n} Q_{8}\right)\right)=3$.

Proof. For $\alpha, \beta \in \Gamma\left(Z_{n} Q_{8}\right)$, let $\alpha=x_{1}+x_{2} a+x_{3} a^{2}+x_{4} a^{3}+x_{5} b+x_{6} a b+$ $x_{7} a^{2} b+x_{8} a^{3} b$ and $\beta=y_{1}+y_{2} a+y_{3} a^{2}+y_{4} a^{3}+y_{5} b+y_{6} a b+y_{7} a^{2} b+y_{8} a^{3} b$.

Case 1 Assume that $p^{i}\left|\left(x_{2}, x_{4}, x_{5}, x_{6}, x_{7}, x_{8}\right), p^{j}\right|\left(y_{2}, y_{4}, y_{5}, y_{6}, y_{7}, y_{8}\right)$ for some $i, j \in\{1,2, \ldots, t-1\}$. Hence, if $i+j \geq t$, then $\alpha-\beta$ is an edge of $\Gamma\left(Z_{n} Q_{8}\right)$. Otherwise, $\alpha-p^{t-j} \alpha-\beta$ is a path of $\Gamma\left(Z_{n} Q_{8}\right)$.

Case 2 Assume that $p \nmid\left(x_{2}, x_{4}, x_{5}, x_{6}, x_{7}, x_{8}\right), p \mid\left(y_{2}, y_{4}, y_{5}, y_{6}, y_{7}, y_{8}\right)$. We know $p^{t-1} \alpha \notin \mathcal{Z}\left(Z_{n} Q_{8}\right)$. Then $\alpha-p^{t-1} \alpha-\beta$ is a path of $\Gamma\left(Z_{n} Q_{8}\right)$.

Case 3 Assume that $p \mid\left(x_{2}, x_{4}, x_{5}, x_{6}, x_{7}, x_{8}\right), p \nmid\left(y_{2}, y_{4}, y_{5}, y_{6}, y_{7}, y_{8}\right)$. We know $p^{t-1} \beta \notin \mathcal{Z}\left(Z_{n} Q_{8}\right)$. Then $\alpha-p^{t-1} \beta-\beta$ is a path of $\Gamma\left(Z_{n} Q_{8}\right)$.

Case 4 Assume that $p \nmid\left(x_{2}, x_{4}, x_{5}, x_{6}, x_{7}, x_{8}\right), p \nmid\left(y_{2}, y_{4}, y_{5}, y_{6}, y_{7}, y_{8}\right)$, then $p^{t-1} \alpha, p^{t-1} \beta \notin \mathcal{Z}\left(Z_{n} Q_{8}\right)$. Then $\alpha-p^{t-1} \alpha-p^{t-1} \beta-\beta$ is a path of $\Gamma\left(Z_{n} Q_{8}\right)$.

Therefore, $\Gamma\left(Z_{n} Q_{8}\right)$ is a connected graph and $\operatorname{diam}\left(\Gamma\left(Z_{n} Q_{8}\right)\right) \leq 3$. In addition, note that $a, b \in \Gamma\left(Z_{n} Q_{8}\right)$, suppose $\gamma=z_{1}+z_{2} a+z_{3} a^{2}+z_{4} a^{3}+z_{5} b+$ $z_{6} a b+z_{7} a^{2} b+z_{8} a^{3} b \in \Gamma\left(Z_{n} Q_{8}\right)$ such that $a \gamma=\gamma a$ and $b \gamma=\gamma b$. Since $a \gamma=\gamma a \Longleftrightarrow z_{6} \equiv z_{8}\left(\bmod p^{t}\right)$ and $z_{5} \equiv z_{7}\left(\bmod p^{t}\right)$ while $b \gamma=\gamma b \Longleftrightarrow z_{2} \equiv z_{4}$ $\left(\bmod p^{t}\right)$ and $z_{6} \equiv z_{8}\left(\bmod p^{t}\right)$, we have $z_{2} \equiv z_{4}\left(\bmod p^{t}\right), z_{5} \equiv z_{7}\left(\bmod p^{t}\right)$ and $z_{6} \equiv z_{8}\left(\bmod p^{t}\right)$. By Lemma 2.2, we know $\gamma \in \mathcal{Z}\left(Z_{n} Q_{8}\right)$. Hence, there does not exist a vertex $\gamma$ of $\Gamma\left(Z_{n} Q_{8}\right)$ such that $a-\gamma-b$ is a path of $\Gamma\left(Z_{n} Q_{8}\right)$. Hence, $\operatorname{diam}\left(\Gamma\left(Z_{n} Q_{8}\right)\right)=3$. 
Lemma 2.4 ([7, Lemma 7.4.9]). Let $F$ be a field of characteristic different from 2. Then

$$
F Q_{8} \cong F \oplus F \oplus F \oplus F \oplus H(F)
$$

Lemma 2.5 ([7, Lemma 7.4.6]). Assume that $\operatorname{char}(F) \neq 2$. Then the quaternion algebra $H(F)$ is either a division ring or is isomorphic to $M_{2}(F)$, the ring of $2 \times 2$ matrices over $F$. The last possibility arises if and only if the equation $X^{2}+Y^{2}=-1$ can be solved in $F$.

Theorem 2.6. Let $p \geq 3$ be a prime. Then $Z_{p} Q_{8} \cong Z_{p} \oplus Z_{p} \oplus Z_{p} \oplus Z_{p} \oplus M_{2}\left(Z_{p}\right)$.

Proof. We know that the equation $X^{2}+Y^{2}=-1$ can always be solved in $Z_{p}$. Owing to Lemma 2.4 and Lemma 2.5, the result follows.

Theorem 2.7. If $p \geq 3$ is a prime, then $\Gamma\left(Z_{p} Q_{8}\right)$ is a graph with $p^{2}+p+1$ connected components of size $p^{4}\left(p^{2}-p\right)$ which each of them is a complete graph.

Proof. By Lemma 2.4, we have $Z_{p} Q_{8} \cong Z_{p} \oplus Z_{p} \oplus Z_{p} \oplus Z_{p} \oplus M_{2}\left(Z_{p}\right)$. For $\alpha=\left(\alpha_{1}, \alpha_{2}, \alpha_{3}, \alpha_{4}, \alpha_{5}\right), \beta=\left(\beta_{1}, \beta_{2}, \beta_{3}, \beta_{4}, \beta_{5}\right) \in \Gamma\left(Z_{p} Q_{8}\right), \alpha_{i}, \beta_{i} \in Z_{p}, \quad i=$ $1,2,3,4$, and $\alpha_{5}, \beta_{5} \in M_{2}\left(Z_{p}\right)$, we can easily conclude that $\alpha_{5} \neq 0, \beta_{5} \neq 0$. If $\alpha_{5}$ and $\beta_{5}$ are not in the same connected component of $M_{2}\left(Z_{p}\right)$, then there is no edge between $\alpha$ and $\beta$. By Lemma 2.1, we know that $\Gamma\left(M_{2}\left(Z_{p}\right)\right)$ is a graph with $p^{2}+p+1$ connected components of size $p^{2}-p$ which each of them is a complete graph. Hence, $\Gamma\left(Z_{p} Q_{8}\right)$ is a graph with $p^{2}+p+1$ connected components of size $p^{4}\left(p^{2}-p\right)$ which each of them is a complete graph.

Theorem 2.8. $\Gamma\left(Z_{2} Q_{8}\right)$ is a graph with 7 connected components of size 32 which each of them is a complete graph.

Proof. First, we construct 7 subsets of $\Gamma\left(Z_{2} Q_{8}\right)$ as below:

$$
A_{1}=\left\{\alpha=x_{1}+x_{2} a+x_{3} a^{2}+x_{4} a^{3}+x_{5} b+x_{6} a b+x_{7} a^{2} b+x_{8} a^{3} b \in \Gamma\left(Z_{2} Q_{8}\right) \mid\right.
$$

$\left.x_{2} \equiv x_{4}(\bmod 2), x_{5} \equiv x_{7}(\bmod 2), x_{i} \in Z_{2}\right\}$.

$A_{2}=\left\{\alpha=x_{1}+x_{2} a+x_{3} a^{2}+x_{4} a^{3}+x_{5} b+x_{6} a b+x_{7} a^{2} b+x_{8} a^{3} b \in \Gamma\left(Z_{2} Q_{8}\right) \mid\right.$

$\left.x_{2} \equiv x_{4}(\bmod 2), x_{6} \equiv x_{8}(\bmod 2), x_{i} \in Z_{2}\right\}$.

$A_{3}=\left\{\alpha=x_{1}+x_{2} a+x_{3} a^{2}+x_{4} a^{3}+x_{5} b+x_{6} a b+x_{7} a^{2} b+x_{8} a^{3} b \in \Gamma\left(Z_{2} Q_{8}\right) \mid\right.$

$\left.x_{5} \equiv x_{7}(\bmod 2), x_{6} \equiv x_{8}(\bmod 2), x_{i} \in Z_{2}\right\}$.

$A_{4}=\left\{\alpha=x_{1}+x_{2} a+x_{3} a^{2}+x_{4} a^{3}+x_{5} b+x_{6} a b+x_{7} a^{2} b+x_{8} a^{3} b \in \Gamma\left(Z_{2} Q_{8}\right) \mid\right.$

$\left.x_{2} \equiv x_{4}(\bmod 2), x_{5}+x_{6}+x_{7}+x_{8} \equiv 0(\bmod 2), x_{i} \in Z_{2}\right\}$.

$A_{5}=\left\{\alpha=x_{1}+x_{2} a+x_{3} a^{2}+x_{4} a^{3}+x_{5} b+x_{6} a b+x_{7} a^{2} b+x_{8} a^{3} b \in \Gamma\left(Z_{2} Q_{8}\right) \mid\right.$

$\left.x_{5} \equiv x_{7}(\bmod 2), x_{2}+x_{4}+x_{6}+x_{8} \equiv 0(\bmod 2), x_{i} \in Z_{2}\right\}$.

$A_{6}=\left\{\alpha=x_{1}+x_{2} a+x_{3} a^{2}+x_{4} a^{3}+x_{5} b+x_{6} a b+x_{7} a^{2} b+x_{8} a^{3} b \in \Gamma\left(Z_{2} Q_{8}\right) \mid\right.$

$\left.x_{6} \equiv x_{8}(\bmod 2), x_{2}+x_{4}+x_{5}+x_{7} \equiv 0(\bmod 2), x_{i} \in Z_{2}\right\}$.

$A_{7}=\left\{\alpha=x_{1}+x_{2} a+x_{3} a^{2}+x_{4} a^{3}+x_{5} b+x_{6} a b+x_{7} a^{2} b+x_{8} a^{3} b \in \Gamma\left(Z_{2} Q_{8}\right) \mid\right.$

$x_{5}+x_{6}+x_{7}+x_{8} \equiv 0(\bmod 2), x_{2}+x_{4}+x_{6}+x_{8} \equiv 0(\bmod 2), x_{2}+x_{4}+x_{5}+x_{7} \equiv$ $\left.0(\bmod 2), x_{i} \in Z_{2}\right\}$.

Clearly, $A_{1} \cup A_{2} \cup \cdots \cup A_{7}=Z_{2} Q_{8} \backslash \mathcal{Z}\left(Z_{2} Q_{8}\right), A_{i} \cap A_{j}=\emptyset, \forall i \neq j$ and $\left|A_{1}\right|=\left|A_{2}\right|=\cdots=\left|A_{7}\right|=32$. 
Second, $\forall \alpha=x_{1}+x_{2} a+x_{3} a^{2}+x_{4} a^{3}+x_{5} b+x_{6} a b+x_{7} a^{2} b+x_{8} a^{3} b \in A_{i}$ and $\forall \beta=y_{1}+y_{2} a+y_{3} a^{2}+y_{4} a^{3}+y_{5} b+y_{6} a b+y_{7} a^{2} b+y_{8} a^{3} b \in \Gamma\left(Z_{2} Q_{8}\right)$, we can conclude that $\alpha \beta=\beta \alpha \Longleftrightarrow \beta \in A_{i}$. Moreover, we can conclude that each connected component $A_{i}(i=1,2, \ldots, 7)$ is a complete graph. This completes our proof.

Lemma 2.9 ([9, Proportion 8.1.20]). Let $R$ be a commutative Noetherian ring and let $G$ be an arbitrary group. Then there exist finitely many indecomposable rings $R_{1}, R_{2}, \ldots, R_{n}$ such that $R G \cong R_{1} G \times R_{2} G \times \cdots \times R_{n} G$. In particular, $\mathscr{U}(R G) \cong \mathscr{U}\left(R_{1} G\right) \times \mathscr{U}\left(R_{2} G\right) \times \cdots \times \mathscr{U}\left(R_{n} G\right)$.

Theorem 2.10. Let $p$ be a prime. Then $\Gamma\left(Z_{2 p} Q_{8}\right)$ is a connected graph and $\operatorname{diam}\left(\Gamma\left(Z_{2 p} Q_{8}\right)\right)=3$.

Proof. (1) If $p=2$, by Theorem 2.3, the result follows.

(2) If $p \geq 3$, by Lemma 2.6 and Lemma 2.9 , we have $Z_{2 p} Q_{8} \cong Z_{2} Q_{8} \oplus Z_{p} \oplus$ $Z_{p} \oplus Z_{p} \oplus Z_{p} \oplus M_{2}\left(Z_{p}\right)$. Then $\forall \alpha=\left(\alpha_{1}, \alpha_{2}, \alpha_{3}, \alpha_{4}, \alpha_{5}, \alpha_{6}\right) \in Z_{2 p} Q_{8}$ and $\beta=$ $\left(\beta_{1}, \beta_{2}, \beta_{3}, \beta_{4}, \beta_{5}, \beta_{6}\right) \in Z_{2 p} Q_{8}$, where $\alpha_{1}, \beta_{1} \in Z_{2} Q_{8}, \alpha_{2}, \beta_{2}, \alpha_{3}, \beta_{3}, \alpha_{4}, \beta_{4}, \alpha_{5}, \beta_{5}$ $\in Z_{p}, \alpha_{6}, \beta_{6} \in M_{2}\left(Z_{p}\right)$. By symmetry, we have the following cases to consider.

First, let $A_{1}, A_{2}, \ldots, A_{7}$ are the sets of vertices of the connected components of $\Gamma\left(Z_{2} Q_{8}\right)$. By Lemma 2.1, we know that there are $p^{2}+p+1$ connected components in $\Gamma\left(M_{2}\left(Z_{p}\right)\right)$ and we denotes them as $B_{i}, \quad i=1,2, \ldots, p^{2}+p+1$.

Case 1 Assume that $\alpha_{1} \in \mathcal{Z}\left(Z_{2} Q_{8}\right), \beta_{1} \in \Gamma\left(Z_{2} Q_{8}\right), \alpha_{6} \in \Gamma\left(M_{2}\left(Z_{p}\right)\right), \beta_{6} \in$ $\mathcal{Z}\left(M_{2}\left(Z_{p}\right)\right)$, then $\alpha-\beta$ is an edge of $\Gamma\left(Z_{2 p} Q_{8}\right)$.

Case 2 Assume that $\alpha_{1}, \beta_{1} \in \mathcal{Z}\left(Z_{2} Q_{8}\right), \alpha_{6}, \beta_{6} \in \Gamma\left(M_{2}\left(Z_{p}\right)\right)$. If $\alpha_{6}, \beta_{6} \in$ $B_{i}$ for some $i$, then $\alpha-\beta$ is an edge of $\Gamma\left(Z_{2 p} Q_{8}\right)$. Otherwise, $\left(\alpha_{1}, \alpha_{2}, \alpha_{3}, \alpha_{4}, \alpha_{5}\right.$, $\left.\alpha_{6}\right)-\left(0,0,0,0,0, \alpha_{6}^{\prime}\right)-\left(\beta_{1}, 0,0,0,0,0\right)-\left(\beta_{1}, \beta_{2}, \beta_{3}, \beta_{4}, \beta_{5}, \beta_{6}\right)$ is a path of $\Gamma\left(Z_{2 p} Q_{8}\right)$, where $\alpha_{6}, \alpha_{6}^{\prime} \in B_{i}$.

Case 3 Assume that $\alpha_{1} \in \mathcal{Z}\left(Z_{2} Q_{8}\right), \beta_{1} \in \Gamma\left(Z_{2} Q_{8}\right), \alpha_{6}, \beta_{6} \in \Gamma\left(M_{2}\left(Z_{p}\right)\right)$. By similar argument above, we have the same results.

Case 4 Let $\alpha_{1}, \beta_{1} \in \Gamma\left(Z_{2} Q_{8}\right), \alpha_{6}, \beta_{6} \in \Gamma\left(M_{2}\left(Z_{p}\right)\right)$.

Subcase 4.1 Suppose that $\alpha_{1}, \beta_{1} \in A_{i}, \alpha_{6}, \beta_{6} \in B_{j}$ for some $i, j$, then $\alpha-\beta$ is an edge of $\Gamma\left(Z_{2 p} Q_{8}\right)$.

Subcase 4.2 Suppose that $\alpha_{1}, \beta_{1} \in A_{i}, \alpha_{6} \in B_{j}, \beta_{6} \in B_{k}$ for some $i, j, k$, $j \neq k$, then $\left(\alpha_{1}, \alpha_{2}, \alpha_{3}, \alpha_{4}, \alpha_{5}, \alpha_{6}\right)-\left(\alpha_{1}, 0,0,0,0,0\right)-\left(\beta_{1}, \beta_{2}, \beta_{3}, \beta_{4}, \beta_{5}, \beta_{6}\right)$ is a path of $\Gamma\left(Z_{2 p} Q_{8}\right)$.

Subcase 4.3 Suppose that $\alpha_{1} \in A_{i}, \beta_{1} \in A_{j}, \alpha_{6} \in B_{t}, \beta_{6} \in B_{k}$ for some $i, j, k, t$ and $i \neq j, t \neq k$, then $\left(\alpha_{1}, \alpha_{2}, \alpha_{3}, \alpha_{4}, \alpha_{5}, \alpha_{6}\right)-\left(\alpha_{1}^{\prime}, 0,0,0,0,0\right)-\left(0,0,0,0,0, \beta_{6}^{\prime}\right)$ $\left(\beta_{1}, \beta_{2}, \beta_{3}, \beta_{4}, \beta_{5}, \beta_{6}\right)$ is a path of $\Gamma\left(Z_{2 p} Q_{8}\right)$, where $\alpha_{1}^{\prime} \in A_{i}, \beta_{6}^{\prime} \in B_{k}$.

Therefore, $\Gamma\left(Z_{2 p} Q_{8}\right)$ is a connected graph and $\operatorname{diam}\left(\Gamma\left(Z_{2 p} Q_{8}\right)\right)=3$.

Theorem 2.11. If $n(>1)$ is not a prime, then $\Gamma\left(Z_{n} Q_{8}\right)$ is a connected graph and $\operatorname{diam}\left(\Gamma\left(Z_{n} Q_{8}\right)\right)=3$.

Proof. Let $n=p_{1}^{t_{1}} p_{2}^{t_{2}} \cdots p_{m}^{t_{m}}$ with $m \geq 2$ and $t_{1}, t_{2}, \ldots, t_{m} \geq 1, p_{1}, p_{2}, \ldots, p_{m}$ are distinct primes and $2 \leq p_{1} \leq p_{2} \leq \cdots \leq p_{m}$. 
(1) When $m=1, n=p_{1}^{t_{1}}, t_{1}>1$, by Theorem 2.3, the result follows.

(2) If $n=2 p, p$ is a prime, by Theorem 2.10, the result follows.

(3) We suppose $m>1, n \neq 2 p$. Let $R_{i}$ denotes $Z_{p_{i}^{t_{i}}} Q_{8}$, then by Lemma 2.9, we have $Z_{n} Q_{8} \cong R_{1} \oplus R_{2} \oplus \cdots \oplus R_{m} \triangleq R$. Note that $\forall \alpha=\left(\alpha_{1}, \alpha_{2}, \ldots, \alpha_{m}\right) \in$ $R, \alpha \in \mathcal{Z}(R)$ if and only if $\alpha_{i} \in \mathcal{Z}\left(R_{i}\right), \forall i=1,2, \ldots, m$. So $\forall \alpha=\left(\alpha_{1}, \alpha_{2}, \ldots\right.$, $\left.\alpha_{m}\right) \in \Gamma(R), \beta=\left(\beta_{1}, \beta_{2}, \ldots, \beta_{m}\right) \in \Gamma(R)$, we should consider the following three cases:

Case 1 Assume that $\forall i=1,2, \ldots, m, \alpha_{i} \in \mathcal{Z}\left(R_{i}\right)$ or $\beta_{i} \in \mathcal{Z}\left(R_{i}\right)$, then $\alpha-\beta$ is an edge of $\Gamma(R)$.

Case 2 Assume that there exists $i \in\{1,2, \ldots, m\}$ such that $\alpha_{i} \in \mathcal{Z}\left(R_{i}\right)$ or $\beta_{i} \in \mathcal{Z}\left(R_{i}\right)$. Without loss of generality, we can assume that $\alpha_{i} \in \mathcal{Z}\left(R_{i}\right)$, and take $\gamma_{i} \in R_{i} \backslash \mathcal{Z}\left(R_{i}\right)$ such that $\beta_{i} \gamma_{i}=\gamma_{i} \beta_{i}$, where $\gamma_{i} \neq \beta_{i}$. Set $\gamma=$ $\left(0,0, \ldots, \gamma_{i}, 0, \ldots, 0\right) \in R$, then $\gamma \in \mathcal{Z}(R)$ and $\gamma \neq \alpha, \beta$. So $\alpha-\gamma-\beta$ is an path of $\Gamma(R)$.

Case 3 Assume that $\forall i=1,2, \ldots, m$, neither $\alpha_{i}$ nor $\beta_{i}$ belongs to $\mathcal{Z}\left(R_{i}\right)$. If there exists $\gamma_{i} \in R_{i} \backslash \mathcal{Z}\left(R_{i}\right)$, where $i=1,2, \ldots, m$, such that $\alpha_{i}-\gamma_{i}-\beta_{i}$ is a path of $\Gamma\left(R_{i}\right)$, then we put $\gamma=\left(0,0, \ldots, \gamma_{i}, 0, \ldots, 0\right) \in R$. It is obvious that $\alpha-\gamma-\beta$ is an path of $\Gamma(R)$. Otherwise, taking $\gamma^{\prime}=\left(\alpha_{1}^{\prime}, 0, \ldots, 0\right) \in \Gamma(R)$ with $\alpha_{1} \alpha_{1}^{\prime}=\alpha_{1}^{\prime} \alpha_{1}$ and $\gamma^{\prime \prime}=\left(0, \ldots, 0, \beta_{m}^{\prime}\right) \in \Gamma(R)$ with $\beta_{m} \beta_{m}^{\prime}=\beta_{m}^{\prime} \beta_{m}$, then $\alpha-\gamma^{\prime}-\gamma^{\prime \prime}-\beta$ is an path of $\Gamma(R)$.

Consequently, we must have $\Gamma(R)$ is a connected graph and $\operatorname{diam}(\Gamma(R)) \leq 3$. Furthermore, note that there must exist an odd prime $q$ such that $q \neq p_{i}, \forall i=$ $1,2, \ldots, m$, we have $q a, q b \in \Gamma(R)$, then by the similar argument of Theorem 2.3 , we can conclude that there doesn't exist a vertex $\alpha$ of $\Gamma(R)$ such that $q a-\alpha-q b$ is a path of $\Gamma(R)$. Thus $\operatorname{diam}(\Gamma(R))=3$. This completes the proof.

\section{The maximum degree and the minimum degree of $\Gamma\left(Z_{n} Q_{8}\right)$}

Lemma 3.1 ([8, Exercise 12]). The number of solutions of congruence equation in $x_{1}, x_{2}, \ldots, x_{k}: a_{1} x_{1}+a_{2} x_{2}+\cdots+a_{k} x_{k} \equiv b(\bmod m)$ which $a_{1}, a_{2}, \ldots, a_{k}, b, m$ are integers and $m \geq 1$, is equal to $m^{k-1}\left(a_{1}, a_{2}, \ldots, a_{k}, m\right)$, if $\left(a_{1}, a_{2}, \ldots, a_{k}, m\right)$ |b.

Lemma 3.2. Assume that $n=p^{t}, x_{2}, x_{4}, x_{5}, x_{6}, x_{7}, x_{8} \in\left\{0,1,2, \ldots, p^{t}-1\right\}$, where $t \geq 2, p \geq 2$ is a prime.

(1) Suppose $p \nmid\left(x_{2}-x_{4}, x_{5}-x_{7}, x_{6}-x_{8}\right)$. Then the number of solutions of congruence system $(*)$ in $y_{2}, y_{4}, y_{5}, y_{6}, y_{7}, y_{8}$ is $p^{4 t}$.

(2) Suppose $p^{\tau} \|\left(x_{2}-x_{4}, x_{5}-x_{7}, x_{6}-x_{8}\right)$, where $1 \leq \tau \leq t-1$. Then the number of solutions of congruence system $(*)$ in $y_{2}, y_{4}, y_{5}, y_{6}, y_{7}, y_{8}$ is $p^{4 t+2 \tau}$.

Proof. (1) First of all, since $p \nmid\left(x_{2}-x_{4}, x_{5}-x_{7}, x_{6}-x_{8}\right)$, without loss of generality, we can suppose $p \nmid\left(x_{2}-x_{4}\right)$.

Case 1.1 Assume that $x_{5}-x_{7} \not \equiv 0\left(\bmod p^{t}\right), x_{6}-x_{8} \not \equiv 0\left(\bmod p^{t}\right)$. Since $\left(x_{2}-x_{4}, x_{6}-x_{8}, p^{t}\right)=1$, so by Lemma 3.1 , we know that the number of solutions 
of the equation $(2)$ is $p^{3 t}$. Suppose $y_{2} \equiv y_{s}^{(2)}\left(\bmod p^{t}\right), y_{4} \equiv y_{s}^{(4)}\left(\bmod p^{t}\right)$, $y_{6} \equiv y_{s}^{(6)}\left(\bmod p^{t}\right), y_{8} \equiv y_{s}^{(8)}\left(\bmod p^{t}\right)$ are the solutions of the equation (2), $s=1,2, \ldots, p^{3 t}$. So we have

(4)

$$
\left(x_{6}-x_{8}\right) y_{s}^{(2)}+\left(x_{8}-x_{6}\right) y_{s}^{(4)}+\left(x_{4}-x_{2}\right) y_{s}^{(6)}+\left(x_{2}-x_{4}\right) y_{s}^{(8)} \equiv 0\left(\bmod p^{t}\right) .
$$

Substituting $y_{2} \equiv y_{s}^{(2)}\left(\bmod p^{t}\right), y_{4} \equiv y_{s}^{(4)}\left(\bmod p^{t}\right)$ into the equation (3), and note that $\left(x_{2}-x_{4}, p^{t}\right)=1$, thus the equation (3) in $y_{5}, y_{7}$ has $p^{t}$ solutions, denoted by $y_{5} \equiv y_{m}^{(5)}\left(\bmod p^{t}\right), y_{7} \equiv y_{m}^{(7)}\left(\bmod p^{t}\right), m=1,2, \ldots, p^{t}$. So we have

$$
\left(x_{5}-x_{7}\right) y_{s}^{(2)}+\left(x_{7}-x_{5}\right) y_{s}^{(4)}+\left(x_{4}-x_{2}\right) y_{m}^{(5)}+\left(x_{2}-x_{4}\right) y_{m}^{(7)} \equiv 0\left(\bmod p^{t}\right) .
$$

In addition, note that $x_{5}-x_{7} \not \equiv 0\left(\bmod p^{t}\right), x_{6}-x_{8} \not \equiv 0\left(\bmod p^{t}\right)$, so by equations (4) and (5), we have

$$
\begin{aligned}
& \left(x_{5}-x_{7}\right)\left(x_{6}-x_{8}\right) y_{s}^{(2)}+\left(x_{5}-x_{7}\right)\left(x_{8}-x_{6}\right) y_{s}^{(4)} \\
& +\left(x_{5}-x_{7}\right)\left(x_{4}-x_{2}\right) y_{s}^{(6)}+\left(x_{5}-x_{7}\right)\left(x_{2}-x_{4}\right) y_{s}^{(8)} \equiv 0\left(\bmod p^{t}\right), \\
& \left(x_{6}-x_{8}\right)\left(x_{5}-x_{7}\right) y_{s}^{(2)}+\left(x_{6}-x_{8}\right)\left(x_{7}-x_{5}\right) y_{s}^{(4)} \\
& +\left(x_{6}-x_{8}\right)\left(x_{4}-x_{2}\right) y_{m}^{(5)}+\left(x_{6}-x_{8}\right)\left(x_{2}-x_{4}\right) y_{m}^{(7)} \equiv 0\left(\bmod p^{t}\right) .
\end{aligned}
$$

From the above equations (6) and (7), we get

$$
\begin{aligned}
& \left(x_{6}-x_{8}\right)\left(x_{4}-x_{2}\right) y_{m}^{(5)}-\left(x_{5}-x_{7}\right)\left(x_{4}-x_{2}\right) y_{s}^{(6)} \\
& +\left(x_{6}-x_{8}\right)\left(x_{2}-x_{4}\right) y_{m}^{(7)}+\left(x_{5}-x_{7}\right)\left(x_{2}-x_{4}\right) y_{s}^{(8)} \equiv 0\left(\bmod p^{t}\right) .
\end{aligned}
$$

Since $p \nmid\left(x_{2}-x_{4}\right)$, thus we have

$$
\left(x_{6}-x_{8}\right) y_{m}^{(5)}+\left(x_{7}-x_{5}\right) y_{s}^{(6)}+\left(x_{8}-x_{6}\right) y_{m}^{(7)}+\left(x_{5}-x_{7}\right) y_{s}^{(8)} \equiv 0\left(\bmod p^{t}\right) \text {. }
$$

It follows that $y_{5} \equiv y_{m}^{(5)}\left(\bmod p^{t}\right), y_{6} \equiv y_{s}^{(6)}\left(\bmod p^{t}\right), y_{7} \equiv y_{m}^{(7)}\left(\bmod p^{t}\right)$, $y_{8} \equiv y_{s}^{(8)}\left(\bmod p^{t}\right)$ satisfy the equation (1). Consequently,

$$
\begin{aligned}
& y_{2} \equiv y_{s}^{(2)} \quad\left(\bmod p^{t}\right), y_{4} \equiv y_{s}^{(4)} \quad\left(\bmod p^{t}\right), y_{5} \equiv y_{m}^{(5)} \quad\left(v p^{t}\right), \\
& y_{6} \equiv y_{s}^{(6)} \quad\left(\bmod p^{t}\right), y_{7} \equiv y_{m}^{(7)} \quad\left(\bmod p^{t}\right), y_{8} \equiv y_{s}^{(8)} \quad\left(\bmod p^{t}\right)
\end{aligned}
$$

are solutions of the system $(*)$.

Therefore, the number of solutions of the system $(*)$ is $p^{3 t} \times p^{t}=p^{4 t}$.

Case 1.2 Assume that $x_{5}-x_{7} \equiv 0\left(\bmod p^{t}\right), x_{6}-x_{8} \neq \equiv 0\left(\bmod p^{t}\right)$. With the same argument of Case 1.1, we know that the equation (2) has $p^{3 t}$ solutions. Moreover, note that $p \nmid\left(x_{2}-x_{4}\right)$, so the equation (3) has and only has $p_{t}$ solutions, i.e., $y_{5} \equiv y_{7} \equiv 0,1, \ldots, p^{t}-1\left(\bmod p^{t}\right)$, and all of them satisfy the equation (1). Hence, the system (*) has and only has $p^{3 t} \times p^{t}=p^{4 t}$ solutions.

Similarly, if $x_{5}-x_{7} \not \equiv 0\left(\bmod p^{t}\right), x_{6}-x_{8} \equiv 0\left(\bmod p^{t}\right)$, we also have the same result. 
Case 1.3 Assume that $x_{5}-x_{7} \equiv 0\left(\bmod p^{t}\right), x_{6}-x_{8} \equiv 0\left(\bmod p^{t}\right)$. Notice that $p \nmid\left(x_{2}-x_{4}\right)$, thus $y_{6} \equiv y_{8} \equiv 0,1, \ldots, p^{t}-1\left(\bmod p^{t}\right)$ and $y_{2} \equiv y_{4} \equiv 0,1, \ldots, p^{t}-1\left(\bmod p^{t}\right)$ satisfy the equation $(2)$. And $y_{5} \equiv y_{7} \equiv$ $0,1, \ldots, p^{t}-1\left(\bmod p^{t}\right)$ and $y_{2} \equiv y_{4} \equiv 0,1, \ldots, p^{t}-1\left(\bmod p^{t}\right)$ satisfy the equation $(3)$. Thus $y_{2} \equiv y_{4} \equiv 0,1, \ldots, p^{t}-1 \quad\left(\bmod p^{t}\right), y_{5} \equiv y_{7} \equiv$ $0,1, \ldots, p^{t}-1 \quad\left(\bmod p^{t}\right), y_{6} \equiv y_{8} \equiv 0,1, \ldots, p^{t}-1 \quad\left(\bmod p^{t}\right)$ satisfy the equation $(*)$. Hence, the system $(*)$ has $p^{2 t} \times p^{t} \times p^{t}=p^{4 t}$ solutions.

(2) We will consider it from two cases:

Case 2.1 Suppose $x_{2}-x_{4}, x_{5}-x_{7}, x_{6}-x_{8} \not \equiv 0\left(\bmod p^{t}\right)$. Since $p^{\tau} \|\left(x_{2}-\right.$ $\left.x_{4}, x_{5}-x_{7}, x_{6}-x_{8}\right)$, without loss of generality, we assume that $x_{2}-x_{4}=p^{\tau} u$, $x_{5}-x_{7}=p^{\lambda} v, x_{6}-x_{8}=p^{\sigma} w$, where $p \nmid u, v, w$ and $t-1 \geq \sigma \geq \lambda \geq \tau \geq 1$. Since $\left(x_{2}-x_{4}, x_{6}-x_{8}, p^{t}\right)=\left(p^{\tau} u, p^{\sigma} w, p^{t}\right)=p^{\tau}$, thus by Lemma 3.1, the total number of solutions of the equation (2) is $p^{3 t} \times p^{\tau}=p^{3 t+\tau}$. Suppose $y_{2} \equiv y_{s}^{(2)}\left(\bmod p^{t}\right), y_{4} \equiv y_{s}^{(4)}\left(\bmod p^{t}\right), y_{6} \equiv y_{s}^{(6)}\left(\bmod p^{t}\right), y_{8} \equiv y_{s}^{(8)}\left(\bmod p^{t}\right)$ are the solutions of the equation $(2), s=1,2, \ldots, p^{3 t+\tau}$. So we have

$$
p^{\sigma} w y_{s}^{(2)}-p^{\sigma} w y_{s}^{(4)}-p^{\tau} u y_{s}^{(6)}+p^{\tau} u y_{s}^{(8)} \equiv 0\left(\bmod p^{t}\right) .
$$

Substituting $y_{2} \equiv y_{s}^{(2)}\left(\bmod p^{t}\right), y_{4} \equiv y_{s}^{(4)}\left(\bmod p^{t}\right)$ into the equation $(3)$, then we will conclude the following equation:

$$
p^{\tau} u y_{5}-p^{\tau} u y_{7} \equiv p^{\lambda} v y_{s}^{(2)}+p^{\lambda} v y_{s}^{(4)}\left(\bmod p^{t}\right) .
$$

Since $\left(p^{\tau}, p^{t}\right)=p^{\tau}$ and $p^{\tau} \mid p^{\lambda}$, thus the equation (3) in $y_{5}, y_{7}$ has $p^{t} \times$ $p^{\tau}=p^{t+\tau}$ solutions. And we denote the solutions as $y_{5} \equiv y_{m}^{(5)}\left(\bmod p^{t}\right)$, $y_{7} \equiv y_{m}^{(7)}\left(\bmod p^{t}\right)$, where $m=1,2, \ldots, p^{t+\tau}$. So we have

$$
p^{\lambda} v y_{s}^{(2)}-p^{\lambda} v y_{s}^{(4)}+p^{\tau} u y_{m}^{(5)}+p^{\tau} u y_{m}^{(7)} \equiv 0\left(\bmod p^{t}\right) .
$$

Moreover, notice that $v, w \neq 0$, so by the equations (8) and (10), we have

$$
\begin{aligned}
& p^{\sigma} w v y_{s}^{(2)}-p^{\sigma} w v y_{s}^{(4)}-p^{\tau} u v y_{s}^{(6)}+p^{\tau} u v y_{s}^{(8)} \equiv 0\left(\bmod p^{t}\right), \\
& p^{\lambda} v w y_{s}^{(2)}-p^{\lambda} v w y_{s}^{(4)}+p^{\tau} u w y_{m}^{(5)}+p^{\tau} u w y_{m}^{(7)} \equiv 0\left(\bmod p^{t}\right) .
\end{aligned}
$$

Furthermore, we can get

$$
\begin{aligned}
& p^{\lambda-\tau+\sigma} w v y_{s}^{(2)}-p^{\lambda-\tau+\sigma} w v y_{s}^{(4)}-p^{\lambda} u v y_{s}^{(6)}+p^{\lambda} u v y_{s}^{(8)} \equiv 0\left(\bmod p^{t}\right) \\
& p^{\sigma-\tau+\lambda} v w y_{s}^{(2)}-p^{\sigma-\tau+\lambda} v w y_{s}^{(4)}+p^{\sigma} u w y_{m}^{(5)}+p^{\sigma} u w y_{m}^{(7)} \equiv 0\left(\bmod p^{t}\right) .
\end{aligned}
$$

So by the equations (13) and (14), we have

$$
p^{\sigma} u w y_{m}^{(5)}-p^{\lambda} u v y_{s}^{(6)}-p^{\sigma} u w y_{m}^{(7)}+p^{\lambda} u v y_{s}^{(8)} \equiv 0\left(\bmod p^{t}\right) .
$$

Notice that $p \nmid u$, we get

$$
p^{\sigma} w y_{m}^{(5)}-p^{\lambda} v y_{s}^{(6)}-p^{\sigma} w y_{m}^{(7)}+p^{\lambda} v y_{s}^{(8)} \equiv 0\left(\bmod p^{t}\right) .
$$


Consequently, $y_{5} \equiv y_{m}^{(5)}\left(\bmod p^{t}\right), y_{6} \equiv y_{s}^{(6)}\left(\bmod p^{t}\right), y_{7} \equiv y_{m}^{(7)}\left(\bmod p^{t}\right)$, $y_{8} \equiv y_{s}^{(8)}\left(\bmod p^{t}\right)$ satisfy the equation $(1)$. Thus,

$$
\begin{aligned}
& y_{2} \equiv y_{s}^{(2)}\left(\bmod p^{t}\right), y_{4} \equiv y_{s}^{(4)}\left(\bmod p^{t}\right), y_{5} \equiv y_{m}^{(5)}\left(\bmod p^{t}\right) \\
& y_{6} \equiv y_{s}^{(6)}\left(\bmod p^{t}\right), y_{7} \equiv y_{m}^{(7)}\left(\bmod p^{t}\right), y_{8} \equiv y_{s}^{(8)}\left(\bmod p^{t}\right)
\end{aligned}
$$

are solutions of the system $(*)$.

Therefore, the number of solutions of the system $(*)$ is $p^{3 t+\tau} \times p^{t+\tau}=p^{4 t+2 \tau}$.

Case2.2 If at least one of $x_{2}-x_{4}, x_{5}-x_{7}, x_{6}-x_{8}$ is 0 in $Z_{p^{t}}$, then the similar argument of Case 2.1 can be applied in here.

Theorem 3.3. Suppose $n=p^{t}$ where $p \geq 2$ is a prime and $t \geq 2$. $\forall \alpha=$ $x_{1}+x_{2} a+x_{3} a^{2}+x_{4} a^{3}+x_{5} b+x_{6} a b+x_{7} a^{2} b+x_{8} a^{3} b \in \Gamma\left(Z_{n} Q_{8}\right)$.

(1) If $p \nmid\left(x_{2}-x_{4}, x_{5}-x_{7}, x_{6}-x_{8}\right)$, then $d(\alpha)=p^{6 t}-p^{5 t}-1$;

(2) If $p^{\tau} \|\left(x_{2}-x_{4}, x_{5}-x_{7}, x_{6}-x_{8}\right)$, where $1 \leq \tau \leq t-1$, then $d(\alpha)=$ $p^{6 t+2 \tau}-p^{5 t}-1$;

(3) The minimum degree $\delta\left(\Gamma\left(Z_{n} Q_{8}\right)\right)=p^{6 t}-p^{5 t}-1$, while $d\left(\alpha=\delta\left(\Gamma\left(Z_{n} Q_{8}\right)\right)\right.$ if and only if $p \nmid\left(x_{2}-x_{4}, x_{5}-x_{7}, x_{6}-x_{8}\right)$.

(4) The maximum degree $\triangle\left(\Gamma\left(Z_{n} Q_{8}\right)\right)=p^{8 t-2}-p^{5 t}-1$, while $d(\alpha)=$ $\triangle\left(\Gamma\left(Z_{n} Q_{8}\right)\right)$ if and only if $p^{t-1} \|\left(x_{2}-x_{4}, x_{5}-x_{7}, x_{6}-x_{8}\right)$.

Proof. (1) Assume that $p \nmid\left(x_{2}-x_{4}, x_{5}-x_{7}, x_{6}-x_{8}\right)$, then by Lemma 3.2, we have $d(\alpha)=p^{2 t} \cdot p^{4 t}-p^{5 t}-1=p^{6 t}-p^{5 t}-1$.

(2) Assume that $p^{\tau} \|\left(x_{2}-x_{4}, x_{5}-x_{7}, x_{6}-x_{8}\right)$, then by Lemma 3.2 , we have $d(\alpha)=p^{2 t} \cdot p^{4 t+2 \tau}-p^{5 t}-1=p^{6 t+2 \tau}-p^{5 t}-1$.

(3) and (4) follows directly by (1) and (2).

Theorem 3.4. Suppose $n=p$ where $p$ is a prime. $\forall \alpha=x_{1}+x_{2} a+x_{3} a^{2}+$ $x_{4} a^{3}+x_{5} b+x_{6} a b+x_{7} a^{2} b+x_{8} a^{3} b \in \Gamma\left(Z_{n} Q_{8}\right)$.

(1) If $p=2$, then $\triangle\left(\Gamma\left(Z_{2} Q_{8}\right)\right)=\delta\left(\Gamma\left(Z_{2} Q_{8}\right)\right)=31$;

(2) If $p^{\tau} \|\left(x_{2}-x_{4}, x_{5}-x_{7}, x_{6}-x_{8}\right)$, where $1 \leq \tau \leq t-1$, then $\triangle\left(\Gamma\left(Z_{n} Q_{8}\right)\right)=\delta\left(\Gamma\left(Z_{n} Q_{8}\right)\right)=p^{6}-p^{5}-1$.

Proof. (1) Owing to Theorem 2.8, the results follows.

(2) By the condition (1) of Lemma 3.2 for $t=1$, we can conclude that the number of solutions of congruence system $(*)$ in $y_{2}, y_{4}, y_{5}, y_{6}, y_{7}, y_{8}$ is $p^{4}$. Hence, $\triangle\left(\Gamma\left(Z_{n} Q_{8}\right)\right)=\delta\left(\Gamma\left(Z_{n} Q_{8}\right)\right)=d(\alpha)=p^{2} \cdot p^{4}-p^{5}-1=p^{6}-p^{5}-1$.

Remark 3.5. Suppose $n>1$ and $n$ has unique normal decomposition $n=$ $p_{1}^{t_{1}} p_{2}^{t_{2}} \cdots p_{m}^{t_{m}}$ with $m \geq 2, t_{1}, t_{2}, \ldots, t_{m} \geq 1$ and $2 \leq p_{1}<p_{2}<\cdots<p_{m}$ where $p_{1}, p_{2}, \ldots, p_{m}$ are distinct primes. By Lemma 2.9, we have

$$
Z_{n} Q_{8} \cong Z_{p_{1}^{t_{1}}} Q_{8} \oplus Z_{p_{2}^{t_{2}}} Q_{8} \oplus \cdots \oplus Z_{p_{m}^{t_{m}}} Q_{8}
$$

Moreover, we denote this isomorphism by $\psi \cdot \forall \alpha=x_{1}+x_{2} a+x_{3} a^{2}+x_{4} a^{3}+$ $x_{5} b+x_{6} a b+x_{7} a^{2} b+x_{8} a^{3} b \in \Gamma\left(Z_{2} Q_{8}\right)$, let $f_{i}=x_{i}$ and let $f_{i 1}, f_{i 2}, \ldots, f_{i m}$ are the remainder of $f_{i} \bmod p_{1}^{t_{1}}, p_{2}^{t_{2}}, \ldots, p_{m}^{t_{m}}$, respectively. Then $\varphi(\alpha)=$ $\left(\alpha_{1}, \alpha_{2}, \ldots, \alpha_{m}\right)$ where $\alpha_{i}=x_{i 1}+x_{i 2} a+x_{i 3} a^{2}+x_{i 4} a^{3}+x_{i 5} b+x_{i 6} a b+x_{i 7} a^{2} b+$ 
$x_{i 8} a^{3} b \in \Gamma\left(Z_{p_{\lambda}{ }_{\lambda}} Q_{8}\right), \lambda=1,2, \ldots, m$. By ([11], Remark 3.6), we have the following results:

(1) let $q^{\sigma}$ denotes any term of $p_{1}^{t_{1}}, p_{2}^{t_{2}}, \ldots, p_{m}^{t_{m}}$, then we can claim that if there exists $1 \leq \tau \leq \sigma-1$ such that $q^{\tau} \mid f_{i}$, then we must have $q^{\tau} \mid f_{i s}$ where $f_{i s}$ is the remainder of $f_{i} \bmod q^{\sigma}$.

(2) If $q^{\tau} \| f_{i}$, then we also have $q^{\tau} \mid f_{i s}$.

Corollary 3.6. Suppose $n$ has at least two distinct prime divisors and the normal decomposition of $n$ and $\alpha$ have been given in Remark 3.5. Let $A_{\lambda}=$ $\left\{\beta \in p_{\lambda}^{t_{\lambda}} \mid \alpha_{\lambda} \beta=\beta \alpha_{\lambda}\right\}, \lambda=1,2, \ldots, m$.

(1) Assume that $t_{\lambda}=1, p_{\lambda}=2$. Then $\left|A_{\lambda}\right|=32$.

(2) Assume that $t_{\lambda}=1, p_{\lambda} \geq 3$.

Then $\left|A_{\lambda}\right|= \begin{cases}p_{\lambda}^{6} & p_{\lambda} \nmid\left(x_{2}-x_{4}, x_{5}-x_{7}, x_{6}-x_{8}\right) \\ p_{\lambda}^{8} & p_{\lambda} \mid\left(x_{2}-x_{4}, x_{5}-x_{7}, x_{6}-x_{8}\right) .\end{cases}$

(3) Assume that $t_{\lambda} \geq 2, p_{\lambda} \nmid\left(x_{2}-x_{4}, x_{5}-x_{7}, x_{6}-x_{8}\right)$. Then $\left|A_{\lambda}\right|=p_{\lambda}^{6 t_{\lambda}}$.

(4) Assume that $t_{\lambda} \geq 2$, $p_{\lambda}^{\tau_{\lambda}} \|\left(x_{2}-x_{4}, x_{5}-x_{7}, x_{6}-x_{8}\right), 1 \leq \tau_{\lambda} \leq t_{\lambda}-1$. Then $\left|A_{\lambda}\right|=p_{\lambda}^{6 t_{\lambda}+2 \tau_{\lambda}}$.

(5) Assume that $t_{\lambda} \geq 2, p_{\lambda}^{t_{\lambda}} \mid\left(x_{2}-x_{4}, x_{5}-x_{7}, x_{6}-x_{8}\right)$. Then $\left|A_{\lambda}\right|=p_{\lambda}^{8 t_{\lambda}}$.

Theorem 3.7. Suppose $n>1, n \neq p^{\lambda}$ where $p \geq 2$ is a prime and $\lambda \geq 1$. The normal decomposition of $n$ has been given in Remark 3.5. $\forall \alpha=x_{1}+x_{2} a+$ $x_{3} a^{2}+x_{4} a^{3}+x_{5} b+x_{6} a b+x_{7} a^{2} b+x_{8} a^{3} b \in \Gamma\left(Z_{n} Q_{8}\right)$ and we define two subsets $I_{1}, I_{2}$ of $I=\{1,2, \ldots, m\}$ as following:

$I_{1}=\left\{\sigma \in I \mid \exists \tau_{\sigma}, 1 \leq \tau_{\sigma} \leq t_{\sigma}-1\right.$, such that $\left.p_{\sigma}^{\tau_{\sigma}} \|\left(x_{2}-x_{4}, x_{5}-x_{7}, x_{6}-x_{8}\right)\right\}$, $I_{2}=\left\{\lambda \in I\left|p_{\lambda}^{\tau_{\lambda}}\right|\left(x_{2}-x_{4}, x_{5}-x_{7}, x_{6}-x_{8}\right)\right\}$. Then

(1) Assume that $p_{i} \neq 2$. Then $d(\alpha)=n^{6} \prod_{\sigma \in I_{1}-\{1\}} p_{\sigma}^{2 \tau_{\sigma}} \prod_{\lambda \in I_{2}-\{1\}} p_{\lambda}^{2 \tau_{\lambda}}-$ $n^{5}-1$;

(2) Assume that $p_{1}=2, t_{1}=1$. Then $d(\alpha)=32 n^{6} \prod_{\sigma \in I_{1}-\{1\}} p_{\sigma}^{2 \tau_{\sigma}} \prod_{\lambda \in I_{2}-\{1\}}$ $p_{\lambda}^{2 \tau_{\lambda}}-n^{5}-1$;

(3) Assume that $p_{1}=2, t_{1} \geq 2$. Then $d(\alpha)=n^{6} \prod_{\sigma \in I_{1}} p_{\sigma}^{2 \tau_{\sigma}} \prod_{\lambda \in I_{2}} p_{\lambda}^{2 \tau_{\lambda}}-$ $n^{5}-1$.

Proof. (1) $\forall \alpha, \beta \in Z_{n} Q_{8}, \varphi(\alpha), \varphi(\beta)$ are defined in Remark 3.5. Then

$$
\begin{aligned}
& \alpha \beta=\beta \alpha \\
\Longleftrightarrow & \left(\alpha_{1}, \alpha_{2}, \ldots, \alpha_{m}\right)\left(\beta_{1}, \beta_{2}, \ldots, \beta_{m}\right)=\left(\beta_{1}, \beta_{2}, \ldots, \beta_{m}\right)\left(\alpha_{1}, \alpha_{2}, \ldots, \alpha_{m}\right) \\
\Longleftrightarrow & \alpha_{i} \beta_{i}=\beta_{i} \alpha_{i}, i=1,2, \ldots, m .
\end{aligned}
$$

By Corollary 3.6, and note that $\left|\mathcal{Z}\left(Z_{n} Q_{8}\right)\right|=n^{5}$, we have $d(\alpha)=\prod_{\sigma \in I_{1}-\{1\}}$ $p_{\sigma}^{6 t_{\sigma}+2 \tau_{\sigma}} \prod_{\lambda \in I_{2}-\{1\}} p_{\lambda}^{8 \tau_{\lambda}} \prod_{k \in I-I_{1}-I_{2}-\{1\}} p_{k}^{6 t_{k}}-n^{5}-1=n^{6} \prod_{\sigma \in I_{1}-\{1\}} p_{\sigma}^{2 \tau_{\sigma}}$ $\prod_{\lambda \in I_{2}-\{1\}} p_{\lambda}^{2 \tau_{\lambda}}-n^{5}-1$.

By the similar argument above, we can conclude that the formulas of (2) and (3). 
Theorem 3.8. Suppose $n>1, n \neq p^{\lambda}$ where $p \geq 2$ is a prime and $\lambda \geq 1$. The normal decomposition of $n$ has been given in Remark 3.5. $\forall \alpha=x_{1}+x_{2} a+$ $x_{3} a^{2}+x_{4} a^{3}+x_{5} b+x_{6} a b+x_{7} a^{2} b+x_{8} a^{3} b \in \Gamma\left(Z_{n} Q_{8}\right)$.

(1) Assume that $p_{i} \neq 2$, then $\delta\left(\Gamma\left(Z_{n} Q_{8}\right)\right)=n^{6}-n^{5}-1$ and $\triangle\left(\Gamma\left(Z_{n} Q_{8}\right)\right)=$ $\frac{n^{8}}{p_{2}^{2}}-n^{5}-1$

(2) Assume that $p_{1}=2, t_{1}=1$, then $\delta\left(\Gamma\left(Z_{n} Q_{8}\right)\right)=32 n^{6}-n^{5}-1$ and $\triangle\left(\Gamma\left(Z_{n} Q_{8}\right)\right)=\frac{32 n^{8}}{p_{2}^{2}}-n^{5}-1$;

(3) Assume that $p_{1}=2, t_{1} \geq 2$, then $\delta\left(\Gamma\left(Z_{n} Q_{8}\right)\right)=n^{6}-n^{5}-1$ and $\triangle\left(\Gamma\left(Z_{n} Q_{8}\right)\right)=\frac{n^{8}}{p_{2}^{2}}-n^{5}-1$.

Proof. (1) By Theorem 3.7, we have $d(\alpha)=\delta\left(\Gamma\left(Z_{n} Q_{8}\right)\right) \Longleftrightarrow I_{1}=\emptyset$ and $I_{2}=\emptyset$. Thus $\delta\left(\Gamma\left(Z_{n} Q_{8}\right)\right)=n^{6}-n^{5}-1$. Moreover, if $t_{2}=1$, then $d(\alpha)=$ $\triangle\left(\Gamma\left(Z_{n} Q_{8}\right)\right) \Longleftrightarrow I_{1}=\{2\}$ and $I_{2}=\{3,4, \ldots, m\}$. So we derive that

$$
\triangle\left(\Gamma\left(Z_{n} Q_{8}\right)\right)=\frac{n^{8}}{p_{2}^{2}}-n^{5}-1 .
$$

By the similar argument above, we can conclude that the formulas of (2) and (3).

Acknowledgments. The first two authors are supported by the National Natural Science Foundation of China(10971024), the Specialized Research Fund for the Doctoral Program of Higher Education(200802860024) and the Nature Science Foundation of Jiangsu Province(No. BK2010393). The third author is supported by the National Natural Science Foundation of China (11161006) and the Guangxi Natural Science Foundation (2011GXNSFA018139).

\section{References}

[1] A. Abdollahi, Commuting graphs of full matrix rings over finite fields, Linear Algebra Appl. 428 (2008), no. 11-12, 2947-2954.

[2] S. Akbari, M. Ghandehari, M. Hadian, and A. Mohammadian, On commuting graphs of semisimple rings, Linear Algebra Appl. 390 (2004), 345-355.

[3] S. Akbari, A. Mohammadian, H. Radjavi, and P. Raja, On the diameters of commuting graphs, Linear Algebra Appl. 418 (2006), no. 1, 161-176.

[4] S. Akbari and P. Raja, Commuting graphs of some subsets in simple rings, Linear Algebra Appl. 416 (2006), no. 2-3, 1038-1047.

[5] G. Karpilovsky, Unit Group of Classical Rings, Clarendon Press, Oxford, 1988.

[6] T. Y. Lam, A First Course in Noncommutative Rings, Springer Verlag, New York, 1991.

[7] C. P. Milies and S. K. Sehgal, An Introduction to Group Rings, Kluwer Academic Publishers, 2002.

[8] C. D. Pan and C. B. Pan, Elementary Number Theory, Second edition, Beijing University Publishing Company, Beijing, 2005.

[9] D. S. Passman, The Algebraic Structure of Group Rings, Wiley - Interscience, John Wiley Sons, New York, 1977.

[10] G. H. Tang and H. D. Su, The properties of zero-divisors graph of $Z_{n}[i]$, J. Guangxi Norm. Univ. Natur. Sci. Ed. 3 (2007), 32-35.

[11] Y. J. Wei, G. H. Tang, and H. D. Su, The commuting graph of the quaternion algebra over residue classes of integers, Ars Combin. 95 (2010), 113-127. 
JIANLONG ChEN

Department of Mathematics

SOUTHEAST UNIVERSITY

NANJing 210096, P. R. China

E-mail address: jlchen@seu.edu.cn

YANYAN GAO

Department of Mathematics

SOUTHEAST University

NANJing 210096, P. R. China

E-mail address: gyy_318@163.com

GaOhua TAng

School of Mathematical Sciences

GuAngXi Education University

Nanning, Guangxi 530001, P. R. China

E-mail address: tanggaohua@163.com 